\title{
Amyloid-beta uptake by blood monocytes is reduced with ageing and Alzheimer's disease
}

\author{
Si-Han Chen 1,2,3, Ding-Yuan Tian 1,2,3, Ying-Ying Shen (10) ${ }^{1,2,3}$, Yuan Cheng $\mathbb{1}^{1,2,3}$, Dong-Yu Fan ${ }^{1,2,3}$, Hao-Lun Sun ${ }^{1,2,3}$,

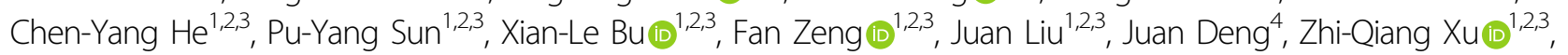 \\ Yang Chen $\mathbb{1}^{1,2,3}$ and Yan-Jiang Wang $\mathbb{1}^{1,2,3,5,6}$
}

\begin{abstract}
Deficits in the clearance of amyloid $\beta$-protein $(A \beta)$ play a pivotal role in the pathogenesis of sporadic Alzheimer's disease (AD). The roles of blood monocytes in the development of AD remain unclear. In this study, we sought to investigate the alterations in the $A \beta$ phagocytosis function of peripheral monocytes during ageing and in $A D$ patients. A total of 104 cognitively normal participants aged 22-89 years, 24 AD patients, 25 age- and sex-matched cognitively normal (CN) subjects, 15 Parkinson's disease patients (PD), and 15 age- and sex-matched CN subjects were recruited. The $A \beta$ uptake by blood monocytes was measured and its alteration during ageing and in $A D$ patients were investigated. $A \beta_{1-42}$ uptake by monocytes decreased during ageing and further decreased in AD but not in PD patients. $A \beta_{1-42}$ uptake by monocytes was associated with $A \beta_{1-42}$ levels in the blood. Among the $A \beta$ uptake-related receptors and enzymes, the expression of Toll-like receptor 2 (TLR2) was reduced in monocytes from AD patients. Our findings suggest that monocytes regulate the blood levels of $A \beta$ and might be involved in the development of $A D$. The recovery of the $A \beta$ uptake function by blood monocytes represents a potential therapeutic strategy for AD.
\end{abstract}

\section{Introduction}

Alzheimer's disease $(\mathrm{AD})$ is the most common neurodegenerative disorder affecting 35 million elderly individuals ${ }^{1}$. Its mechanism remains unclear, and no diseasemodifying therapies are currently available. A large amount of evidence suggests that deficit in the clearance of amyloid $\beta$-protein $(A \beta)$, which leads to the cerebral accumulation of $A \beta$, plays a pivotal role in the development of sporadic $\mathrm{AD}^{2}$.

Recent studies show that a series of $\mathrm{AD}$ risk gene mutations are associated with immune responses and endocytosis, and these include ATP-binding cassette transporter A7, CD33, triggering receptor expressed on myeloid cells-2

\footnotetext{
Correspondence: Yang Chen (cytgzy2020@163.com) or

Yan-Jiang Wang (yanjiang_wang@tmmu.edu.cn)

'Department of Neurology and Centre for Clinical Neuroscience, Daping

Hospital, Third Military Medical University, Chongqing, China

${ }^{2}$ The Institute of Brain and Intelligence, Third Military Medical University,

Chongqing, China

Full list of author information is available at the end of the article

These authors contributed equally: Si-Han Chen, Ding-Yuan Tian
}

(TREM2) and complement receptor 1. These findings suggest that dysfunction of innate immunity, mainly involving microglia and peripheral myeloid cells, is a critical reason for $\mathrm{AD}^{3-7}$. Indeed, studies have suggested that the reduced $\mathrm{A} \beta$ uptake capacity of microglia in the brain is a major mechanism underlying the development of $\mathrm{AD}^{8,9}$. However, the alterations in the functions and roles of peripheral myeloid cells during ageing and in $\mathrm{AD}$ remains unclear.

While resident microglia play a key role in the clearance of $A \beta$ in the brain, approximately $40-60 \%$ of $A \beta$ generated in the brain is estimated to diffuse into the blood and be cleared in the periphery, implying that the peripheral system also plays an essential role in clearing $A \beta$ from the brain ${ }^{10-12}$. It remains undetermined how this brain-derived $A \beta$ is cleared in the periphery. Blood monocytes are the counterparts of microglia in the periphery. Some studies have demonstrated that monocytes are more effective at neuroprotection, neuroinflammation regulation and $A \beta$ clearance than microglia in $A D^{13-17}$. Therefore, monocytes might play a critical role in the clearance of brain-derived $A \beta$ in the periphery.

\section{(c) The Author(s) 2020}

(c) Open Access This article is licensed under a Creative Commons Attribution 4.0 International License, which permits use, sharing, adaptation, distribution and reproduction cc) in any medium or format, as long as you give appropriate credit to the original author(s) and the source, provide a link to the Creative Commons license, and indicate if changes were made. The images or other third party material in this article are included in the article's Creative Commons license, unless indicated otherwise in a credit line to the material. If material is not included in the article's Creative Commons license and your intended use is not permitted by statutory regulation or exceeds the permitted use, you will need to obtain permission directly from the copyright holder. To view a copy of this license, visit http://creativecommons.org/licenses/by/4.0/. 
In the present study, we aimed to investigate alterations in $A \beta$ uptake by peripheral monocytes during ageing in cognitively normal $(\mathrm{CN})$ subjects and in sporadic $\mathrm{AD}$ patients and to evaluate the role of peripheral monocytes in the uptake of $A \beta$ in the blood.

\section{Methods}

\section{Study subjects}

A total of $104 \mathrm{CN}$ participants aged $22-89$ years, $24 \mathrm{AD}$ patients, 25 age- and sex-matched CN subjects, 15 Parkinson's disease (PD) patients and 15 age- and sexmatched $\mathrm{CN}$ subjects were recruited from Daping Hospital between March 2017 and May 2019. Another 25 $\mathrm{CN}$ subjects were enrolled for plasma collection. Based on our preliminary data, a minimum sample size of 13 patients and $13 \mathrm{CNs}$ were estimated to be necessary to show a difference in $A \beta$ uptake abilities by monocytes between $\mathrm{AD}$ patients and $\mathrm{CNs}$ with a power of $80 \%$ and an $\alpha$ of $5 \%$.

Subjects were not eligible if they had a family history of dementia; had a concomitant neurologic disorder except for $\mathrm{AD}$ and $\mathrm{PD}$; were in a state of obvious infection or inflammation potentially affecting the status of blood cells; had severe cardiac, pulmonary, hepatic, renal diseases or any kinds of tumour; had any potent haematopathy, including acute monocytic leukaemia and myelodysplastic syndrome, during the recovery period of agranulocytosis; had autoimmune diseases, including rheumatoid arthritis and systemic lupus erythematosus; had an endocrine system disease, including Cushing syndrome and thyroid disorders; and declined to participate in the study.

The study was approved by the ethics committee of Daping Hospital. Written consent was obtained from participants or their legal representatives.

\section{Clinical assessment}

The clinical evaluation was performed by following the protocol described in our previous studies ${ }^{18}$. In brief, demographic data including age, sex, education level and occupation were collected on admission. The medical history, including current medications, prior head trauma and surgery, prior gas poisoning, schizophrenia, hypothyroidism, coronary heart diseases, atrial fibrillation, cerebrovascular diseases, chronic obstructive pulmonary disease, chronic hepatitis, chronic renal insufficiency, hypertension, diabetes mellitus, hypercholesterolaemia and regular use of non-steroidal anti-inflammatory or prescription drugs, was collected from the medical records and a formal questionnaire.

Cognitive status was assessed using a neuropsychological battery that included Minimum Mental State Examination (MMSE), Activities of Daily Living and Montreal Cognitive Assessment (MoCA). Subjects with abnormal performance in MMSE or MoCA assessment were further subjected to neuropsychological tests, including Clinical Dementia Rating, Pfeiffer Outpatient Disability Questionnaire and Hachinski Ischaemic Score (for assessing significant vascular diseases). Subjects with abnormal cognition were further subjected to a brain computed tomography/magnetic resonance imaging investigation and blood tests for thyroxine, vitamin B12, folic acid and HIV/syphilis to rule out metabolic and infectious reasons for cognitive decline.

Dementia was diagnosed based on Diagnostic and Statistical Manual of Mental Disorders, Fourth Edition criteria. The diagnosis of probable $\mathrm{AD}$ was made according to the criteria of the National Institute of Neurological and Communicative Diseases and Stroke and the Alzheimer Disease and Related Disorders Association. Idiopathic PD was diagnosed according to the Parkinson's Disease Society Brain Bank criteria ${ }^{19}$.

\section{Blood sampling}

To avoid possible circadian rhythm effects, the sampling conditions, including sampling timing and fasting state, were consistent among $\mathrm{AD}$ and $\mathrm{PD}$ patients and their matched $\mathrm{CN}$ subjects. A portion of fasting blood samples was aliquoted for measuring complete blood cell counts, fasting glucose, thyroxin, creatinine, urea, uric acid, aspartate aminotransferase, alanine aminotransferase and total cholesterol levels. For another portion of blood, plasma was separated within $30 \mathrm{~min}$ after sampling and stored at $-80^{\circ} \mathrm{C}$ for further analysis of $\mathrm{A} \beta$. For $\mathrm{A} \beta$ uptakerelated assay, the blood samples were applied for the isolation of peripheral blood mononuclear cells (PBMCs) within $2 \mathrm{~h}$ after blood draw.

\section{Isolation of blood monocytes}

Fresh heparinized blood was diluted with phosphatebuffered saline (PBS; 1:1 ratio; vol/vol). PBMCs were isolated by density gradient centrifugation using FicollHypaque (TBD Science, Tianjin, China), and mononuclear sections were collected and washed with PBS three times. A portion of the PBMCs was used for the A $\beta$ uptake assay, and the other portion of PBMCs was used for monocyte isolation by CD14 Microbeads (Miltenyi Biotec, Bergisch Gladbach, Germany) and passed through a magnetic activated cell sorting (MACS) column for the positive selection of $\mathrm{CD} 14^{+}$cells, according to the manufacturer's instructions. The remaining PBMCs were frozen at a concentration of $1-2 \times 10^{6}$ cells $/ \mathrm{ml}$ in $10 \%$ dimethyl sulfoxide (Sigma-Aldrich, Saint Louis, USA)/90\% foetal calf serum (vol/vol Gibco, CA, USA) for future use.

\section{$A \beta$ uptake assay}

Isolated PBMCs were resuspended in RPMI medium with $10 \%$ foetal calf serum and $1 \%$ penicillin/streptomycin 
and adjusted to a concentration of $2 \times 10^{6}$ cells $/ \mathrm{ml}$. To test the uptake of $A \beta$, PBMCs were incubated with FITC-A $\beta_{1-42}(2 \mu \mathrm{g} / \mathrm{ml})$ (GL Biochem, Shanghai, China) overnight at $37^{\circ} \mathrm{C}$ in a $5 \% \mathrm{CO}_{2}$ incubator. Following incubation, the cell suspensions were discarded, and adherent cells were detached from the well plate by $0.25 \%$ trypsin and washed with fluorescence-activated cell sorting (FACS) buffer twice. Then the cell suspensions were preincubated with Human TruStain FcX (Biolegend, CA, USA) on ice for $20 \mathrm{~min}$ to avoid producing a high background by the non-specific binding of the Fc receptor expressed on immune cells to the Fc fragment of the fluorophore-labelled antibody. Then the cell suspensions were washed and stained with the following fluorophorelabelled antibodies according to the corresponding manufacturer's instructions (BD, NJ, USA): allophycocyanin (APC)-anti human CD14, phycoerythrin (PE)-anti human CD16, APC-mouse IgG2a, $\mathrm{k}$ isotype control, PE-mouse IgG2a, and $\mathrm{k}$ isotype control. Following incubation, the cells were washed twice with FACS buffer and fixed with $1 \%$ paraformaldehyde. Flow cytometry was performed on a NovoCyte Flow Cytometer (ACEA Biosciences, CA, USA) after appropriate compensation. Monocytes were gated using forward and side scatter, and monocyte subsets were identified by differential expression of CD14 and CD16 (Fig. 1G, H). The data were analysed by the NovoExpress software based on forward and side scatter and the mean fluorescence intensity. To maintain consistent testing conditions, a gating strategy was designed and applied equivalently across all study samples.

\section{Imaging flow cytometry (IFC)}

IFC was performed according to a previous report ${ }^{20}$. In brief, the procedures for labelling surface markers were the same as those used for conventional flow cytometry, which was described above. IFC was performed on a twocamera ISX with the INSPIRE acquisition software (Amnis, NJ, USA). Excitation lasers used for analysis included $5 \mathrm{~mW} 405 \mathrm{~nm}, 100 \mathrm{~mW} 488 \mathrm{~nm}$ and $150 \mathrm{~mW}$ $642 \mathrm{~nm}$. A $2.5 \mathrm{~mW} 785 \mathrm{~nm}$ laser was used for internal calibration to provide a scatter signal and measure speed beads. FITC and PE were excited by the $488 \mathrm{~nm}$ laser, and the emission was captured in the ranges of $505-560 \mathrm{~nm}$ (Ch02) and $560-595 \mathrm{~nm}$ (Ch03). APC was excited by the $647 \mathrm{~nm}$ laser, and the emission was captured in the wavelength range of 642-745 nm (Ch05). In total, 25,000 events were acquired, and all images were captured with the $\times 20$ objective and a cell classifier (threshold) applied to the bright field channel (Ch01) to exclude small particles (Fig. 1A, B). Monocytes were identified using the Amnis IDEAS software. Cells with high-intensity labelling of the CD14 marker were chosen as monocytes (Fig. 1CR3). For analysis of $A \beta_{1-42}$ internalization feature, highintensity labelling of FITC-conjugated $A \beta_{1-42}$ monocytes were chosen, following selection of single cells with highintensity labelling of APC-conjugated CD14 (Supplemental Fig. $1 \mathrm{a}-\mathrm{C}$ ). The internalization of FITC-A $\beta_{1-42}$ by monocytes is defined as the ratio of the intensity inside the cell to the intensity of the entire cell. Internalized cells typically have positive scores while cells with little internalization have negative scores. Cells with scores around 0 have a mix of internalization and membrane intensity. Followed by gating on internalized monocytes with positive score and negative score, composite of images of channel 2 (FITC-A $\beta_{1-42}$ ) and channel 5 (APC-CD14) are shown (Supplemental Fig. 11d, e).

\section{Confocal microscopy}

Human monocytes were enriched by plastic adhesion overnight and seeded onto a collagen-coated MatTek culture dish with $10 \mu \mathrm{m}$ cover slide on the bottom. For FITC-A $\beta_{1-42}$ uptake, monocytes were incubated with FITC-A $\beta_{1-42}(2 \mu \mathrm{g} /$ $\mathrm{ml}$ ) overnight at $37^{\circ} \mathrm{C}$ in a $5 \% \mathrm{CO}_{2}$ incubator. Cells were washed three times with ice-cold PBS and stained with antihuman CD14 monoclonal antibody (Abcam, Cambridge, Britain) at $4{ }^{\circ} \mathrm{C}$. Cells were washed with PBS and stained with Alexa Fluor 594 Anti-Mouse IgG (Invitrogen, CA, USA) for $1 \mathrm{~h}$ and then mounted with Mounting Medium with 4',6-diamidino-2-phenylindole (Santa Cruz, CA, USA). The dishes were examined by OLYMPUS confocal microscope.

\section{$A \beta_{1-42}$ assay}

Plasma $A \beta_{1-42}$ levels were measured using an ultrasensitive single molecule array (SIMOA) on the Simoa HD-1 Analyser (Quanterix, Lexington, MA), as previously described $^{21}$. SIMOA technique implies immunocapture of the target protein on magnetic beads, which are trapped in femtoliter volume wells, followed by the addition of enzymelabelled detection antibody and accurate digital quantification. The high analytical sensitivity of this technique allows for pre-dilution of plasma samples, thus contributing to reducing matrix interferences. It has been widely used and been validated useful in numerous studies ${ }^{22}$.

\section{Measurement of $A \beta$ uptake-related receptors in monocytes}

The staining of $\mathrm{A} \beta$ uptake-related receptors, including Toll-like receptor 2 (TLR2), TREM2, CD36, CD33 and macrophage scavenger receptor 1 (SCARA1), was performed by flow cytometry. Following CD14-positive selection by MACS, $1.0 \times 10^{5}$ monocytes were preincubated with Human TruStain FcX (Biolegend, CA, USA) on ice for $20 \mathrm{~min}$. For cell surface staining, cells were incubated with monoclonal antibodies against APC-anti human CD33, BB515-anti human CD282, Percp-CyTM5.5-anti human CD36, BV421anti human MSR1 (Biolegend, CA, USA) and PE-anti human TREM2 for $15 \mathrm{~min}$, washed via centrifugation twice and fixed with $1 \%$ paraformaldehyde. Cells were acquired on a FACS 

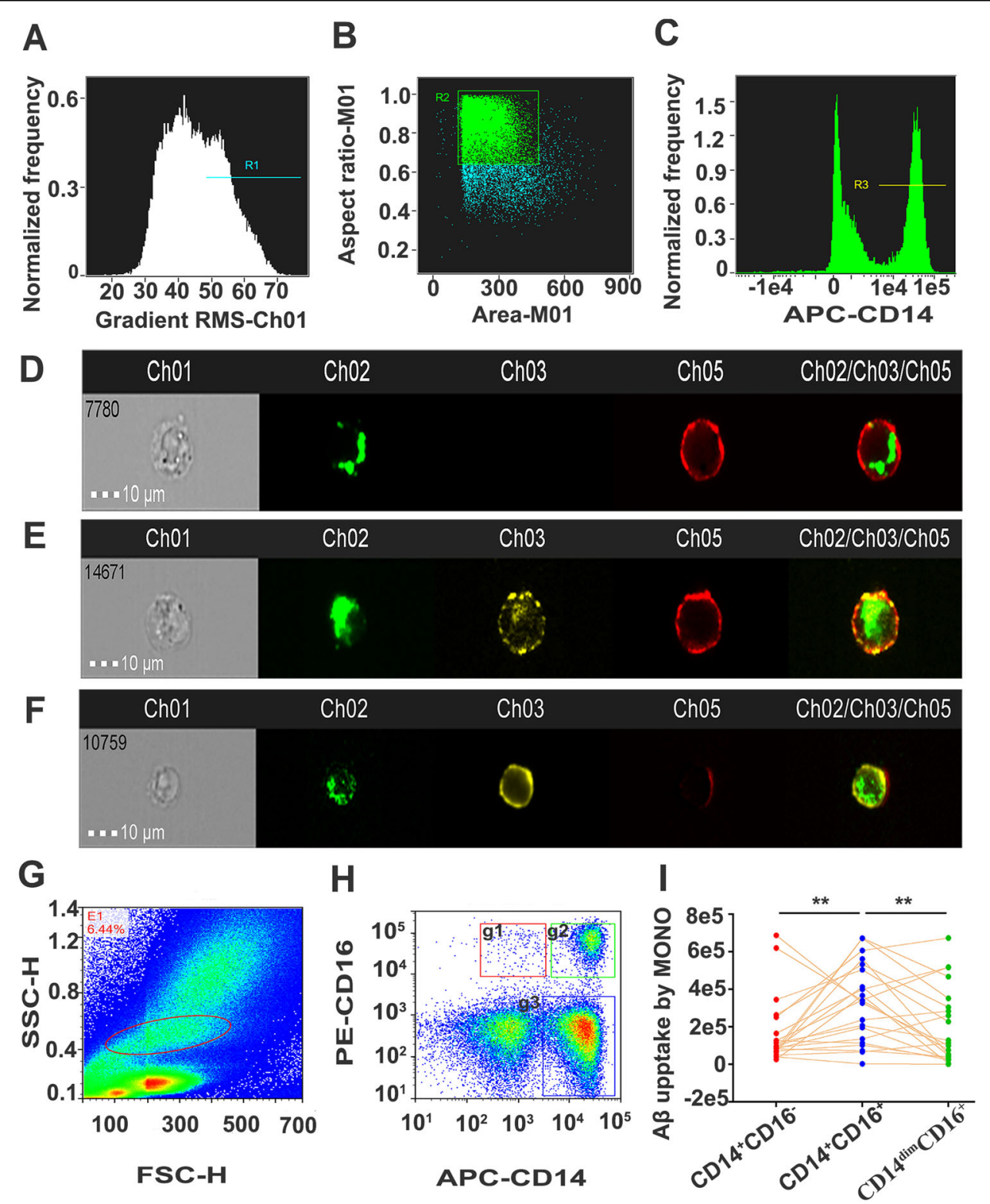

Fig. 1 Imaging flow cytometry of $\mathbf{A} \boldsymbol{\beta}_{1-42}$ uptake of monocyte subsets. A Single cells were selected from debris by gating on cells in focus, B followed by gating on the area and aspect ratio of the brightfield image. C Cells with high-intensity labelling of CD14 were chosen as monocytes. Monocytes were stained with APC-conjugated anti-CD14 mAb (red) and PE-conjugated anti-CD16 mAb (yellow), whereas FITC-conjugated A $\beta_{1-42}$ is shown in green. Images of FITC-labelled $A \beta_{1-42}$ uptake by the classic $C D 14^{+} C D 16^{-}$monocyte subset $(\mathbf{D})$, intermediate $C D 14^{+} C D 16^{+}$monocyte subset $(\mathbf{E})$ and non-classical $C D 14^{\operatorname{dim}} C D 16^{+}$monocyte subset were collected $(\mathbf{F}) . \mathbf{G}, \mathbf{H}$ For the measurement of $A \beta_{1-42}$ uptake by monocyte subsets, monocytes were gated based on FSC-H and SSC-H. Three monocyte subsets, including classical monocyte subset CD $14^{+} \mathrm{CD} 16^{-}(\mathbf{g} \mathbf{1})$, intermediate monocyte subset $\mathrm{CD} 14^{+} \mathrm{CD} 16^{+}$(g2) and non-classical monocyte subset $\mathrm{CD} 14^{\mathrm{dim}} \mathrm{CD} 16^{+}(\mathbf{g} \mathbf{3})$, were gated based on the expression of CD14 and CD16. I $n=44$ per group, a paired $t$ test, two sided. ${ }^{* *} p<0.01$. MONO monocytes, FSC-H forward scatter height, SSC-H side scatter height, A 3 amyloid- $\beta$ protein.

Navios (Beckman, CA, USA), and analyses were performed using the Flowjo v10 software.

\section{Measurement of $A \beta$-degrading enzymes in monocytes}

Western blotting was performed as previously described. PBMCs were lysed in RIPA buffer. Samples were subjected to electrophoresis on sodium dodecyl sulfate-polyacrylamide gel electrophoresis $(8-12 \%$ acrylamide) gels. The blots were probed with antibodies against cathepsin D (1:1000, monoclonal, Arigobio), cathepsin S (1:1000, monoclonal, Arigobio) and $\beta$-actin (1:1000, monoclonal, Sigma-Aldrich). The protein bands 
were scanned using the Odyssey scanner software (LiCOR Bioscience, CA, USA) and quantified by Quantity One 6.0. The band density was normalized to that of $\beta$-actin in the same sample.

\section{Statistical analysis}

For each statistical analysis, appropriate tests were selected based on whether or not the data were normally distributed (D'Agostino-Pearson normality test). Differences in demographic characteristics were assessed by Chi-square test. For normally distributed data, statistical comparisons between two groups were made using Student's $t$ test or paired $t$ test (or Student's $t$ test with Welch's correction if the $F$-test showed significantly different variances between groups). For non-normally distributed data, Wilcoxon matched-pairs test or Mann-Whitney test was used, where appropriate. Specifically, values of monocyte $A \beta$ uptake of $\mathrm{AD}, \mathrm{PD}$ and their age- and sex-matched $\mathrm{CN}$ were logarithmically transformed to an approximate normal distribution. Student's $t$ test was used to determine the differences between groups. A Spearman correlation or covariate correlation analysis was utilized to analyse the association of $A \beta_{1-42}$ uptake with ageing or $A \beta_{1-42}, A \beta_{1-40}$ levels, and $A \beta_{1-42} / A \beta_{1-40}$ ratio in the plasma, respectively. The trajectory of $A \beta_{1-42}$ uptake with age was modelled using Fit spline/Lowess (cubic) spline. All statistical analyses were performed with the GraphPad Prism v5.0 software. The data are expressed as the mean \pm SEM, unless otherwise stated. $P$ values $<0.05$ (two sided) were considered significant.

\section{Results}

\section{$A \beta_{1-42}$ uptake by monocyte subsets}

In humans, peripheral monocytes are divided into three subsets based on the expression of the cell-surface markers CD14 (a pattern recognition receptor for lipopolysaccharide) and CD16 (Fcy III receptor), including non-classic monocytes with low CD14 expression and high CD16 expression $\left(\mathrm{CD} 14^{\mathrm{dim}} \mathrm{CD} 16^{+}\right.$or $\left.\mathrm{CD} 14^{-} \mathrm{CD} 16^{+}\right)$, intermediate monocytes with high or intermediate CD14 and $\mathrm{CD} 16$ expression $\left(\mathrm{CD} 14^{+} \mathrm{CD} 16^{+}\right)$, and classic monocytes with high CD14 expression but very low or negative CD16 expression $\left(\mathrm{CD} 14^{+} \mathrm{CD} 16^{-}\right)^{23}$. We found that all subsets of monocytes could take up $A \beta_{1-42}$ (Fig. 1D-F). Intracellular location of $A \beta_{1-42}$ was further validated by confocal stacks (Supplemental Fig. 1). For IFC, the median internalization erode of FITC-A $\beta_{1-42}$ by monocyte was 1.598 , and the positive rate of $A \beta_{1-42}$ internalized monocytes almost reached $93.1 \%$, indicating that $A \beta$ proteins were taken up by monocytes (Supplemental Figs. 1 and 2). The $\mathrm{CD} 14^{+} \mathrm{CD} 16^{+}$subset had the highest uptake of $\mathrm{A} \beta_{1-42}$ among the three subsets, with no significant difference in $\mathrm{A} \beta_{1-42}$ uptake between the $\mathrm{CD} 14^{+} \mathrm{CD} 16^{+}$and CD14 ${ }^{\text {dim }} \mathrm{CD} 16^{+}$subsets (Fig. 1I).

\section{$A \beta_{1-42}$ uptake by monocyte subsets during ageing}

Then we measured $A \beta_{1-42}$ uptake by monocytes in 104 $\mathrm{CN}$ subjects aged 22-89 years who were not different in sex among the different age groups (Supplemental Table 1). $\mathrm{A} \beta_{1-42}$ uptake by the total monocyte population was correlated with age; that is, the older the age was, the lower the $A \beta_{1-42}$ uptake level $(\gamma=-0.3661, P=0.0001)$. Among the three subsets, $A \beta_{1-42}$ uptake was correlated with age in the $\mathrm{CD}_{14}{ }^{+} \mathrm{CD} 16^{-} \quad(\gamma=-0.374, \quad P<0.0001)$ and $\mathrm{CD} 14{ }^{\mathrm{dim}} \mathrm{CD} 16^{+}$subsets $(\gamma=-0.285, \quad P=0.003)$, but not in the $\mathrm{CD} 14^{+} \mathrm{CD} 16^{+}$subset $(\gamma=-0.100, P=0.311$; Fig. $2 \mathrm{~A}-\mathrm{D}) . \mathrm{A} \beta_{1-42}$ uptake by total monocytes and the $\mathrm{CD} 14^{+} \mathrm{CD} 16^{-}$subset decreased rapidly in the group aged 20-40 years, but the reduction rate became relatively slow after 40 years of age. $\mathrm{A} \beta_{1-42}$ uptake by $\mathrm{CD} 14^{\mathrm{dim}} \mathrm{CD} 16^{+}$ subset decreased rapidly in the group aged $40-60$ years, but the reduction rate became relatively slow after 60 years of age. This result suggests that the decrease in $A \beta$ uptake is a life-long process that may be different among the three monocyte subsets and occurs prior to the cerebral deposition of $\mathrm{A} \beta$ (Fig. 2E).

\section{Correlation between $A \beta_{1-42}$ uptake by monocytes and $A \beta$ levels in the blood}

After controlling the confounding factors of age, sex, vascular risk factors and $A P O E \varepsilon 4$ genotype, the partial correlation analysis showed that $A \beta_{1-42}$ uptake by total monocytes was significantly correlated with plasma $A \beta_{1-42}$ levels $(\gamma=-0.668, P=0.002)$ in $\mathrm{CN}$ subjects $(n=25$, mean age $60.54 \pm 17.21$ years). Among them, $\mathrm{A} \beta_{1-42}$ uptake by both $\mathrm{CD}_{14}{ }^{+} \mathrm{CD} 16^{-} \quad(\gamma=-0.576, \quad P=0.010) \quad$ and $\mathrm{CD} 14{ }^{\operatorname{dim}} \mathrm{CD}_{16}{ }^{+}(\gamma=-0.506, P=0.027)$ subsets, but not the $\mathrm{CD} 14^{+} \mathrm{CD} 16^{+}$subset $(\gamma=-0.390, P=0.099)$, was correlated with plasma $A \beta_{1-42}$ levels (Fig. 3). Moreover, $A \beta_{1-}$ 42 uptake by total monocytes was significantly correlated with plasma $\mathrm{A} \beta_{1-40}$ levels $(\gamma=-0.615, P=0.005$; Fig. 4A). Among them, $\mathrm{A} \beta_{1-42}$ uptake by $\mathrm{CD} 14^{+} \mathrm{CD} 16^{-}(\gamma=-0.640$, $P=0.003)$ and $\mathrm{CD} 14^{+} \mathrm{CD} 16^{+}(\gamma=-0.458, P=0.048)$ subsets, but not the $\mathrm{CD} 14^{\operatorname{dim}} \mathrm{CD} 16^{+}(\gamma=-0.327, P=$ 0.172) subset, was correlated with plasma $A \beta_{1-40}$ levels (Fig. 4B-D). A $\beta_{1-42}$ uptake by CD14 ${ }^{\mathrm{dim}} \mathrm{CD} 16^{+}(\gamma=-0.470$, $P=0.042)$ subset, but not the total monocyte $(\gamma=-0.235$, $P=0.334), \mathrm{CD}_{14}{ }^{+} \mathrm{CD} 16^{-}(\gamma=-0.039, P=0.874)$ and $\mathrm{CD} 14^{+} \mathrm{CD}^{+} 6^{+}(\gamma=-0.080, P=0.746)$ subsets, was correlated with plasma $A \beta_{1-42} / A \beta_{1-40}$ ratio (Supplemental Fig. 3).

\section{$A \beta_{1-42}$ uptake by monocytes from $A D$ and PD patients and $\mathrm{CN}$ subjects}

To investigate whether the alteration in $A \beta_{1-42}$ uptake by monocytes is specific to AD patients, $24 \mathrm{AD}$ patients, 15 ageand sex-matched PD patients and their matched CNs were enrolled (Supplemental Tables 2 and 3). There were no significant differences in sex, age, years of education, $A P O E \& 4$ genotype, comorbidities, including hypertension, 

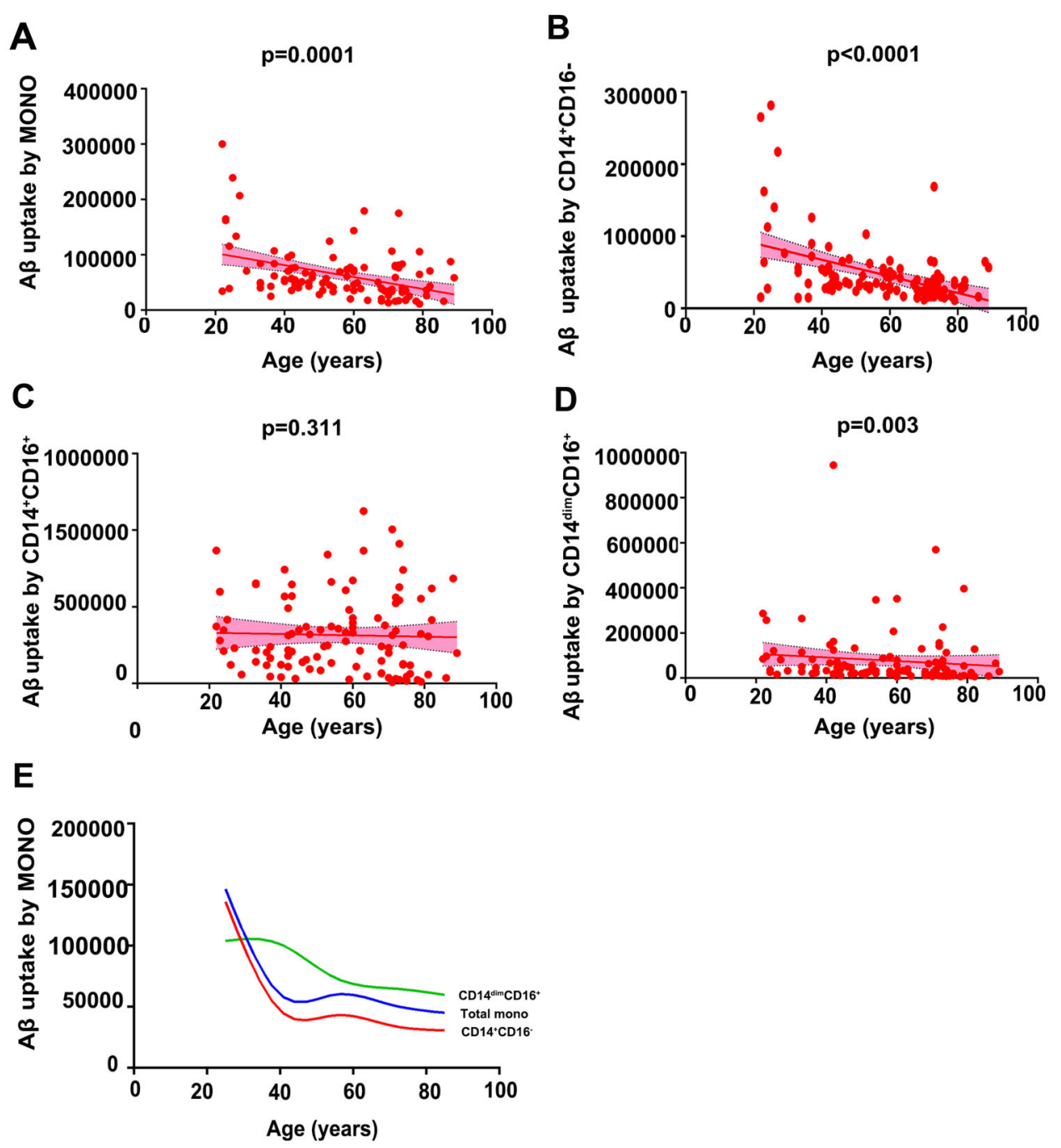

Fig. 2 Correlation and trajectory of $A \beta_{1-42}$ uptake by monocyte subsets relative to age. Spearman correlation analysis was utilized to investigate the correlation between age and the uptake of $A \beta_{1-42}$ by all monocytes $(\mathbf{A})$, by the $C D 14^{+} C D 16^{-}$subset $(\mathbf{B})$, by the $C D 14^{+} C D 16^{+}$subset (C) and by the $\mathrm{CD} 14^{\mathrm{dim}} \mathrm{CD} 16^{-}$subset (D). E The trajectory of $A \beta_{1-42}$ uptake by total monocytes, $\mathrm{CD} 14^{\mathrm{dim}} \mathrm{CD} 16^{+}$and the $\mathrm{CD} 14^{+} \mathrm{CD} 16^{-}$subsets relative to age was modelled using Fit spline/Lowess (cubic) spline. $n=104$. MONO monocytes, $A \beta$ amyloid- $\beta$ protein.

hyperlipidaemia and diabetes mellitus, or medications between the matched groups. We found that $A \beta_{1-42}$ uptake by total monocytes and the various subsets was lower in $A D$ patients than in CNs (Fig. 5). There were no significant differences in $A \beta_{1-42}$ uptake by total monocytes and their subsets between PD patients and CNs (Supplemental Fig. 4). These results suggest that $A \beta_{1-42}$ uptake by monocytes might be specifically decreased in $\mathrm{AD}$ patients.

\section{Expression of $A \beta_{1-42}$ uptake-related receptors and} $A \beta$-degrading enzymes in monocytes of $A D$ patients

In $A D$, we tested five major $A \beta$-uptake receptors involved with myeloid cell-mediated physiological uptake of $A \beta$, including TLR2, TREM2, CD36, CD33 and SCARA1. The expression of TLR2 was lower in AD patients than in CNs.
However, no differences were observed in the expression of receptors, including TREM2, CD36, CD33 and SCARA1, between $\mathrm{AD}$ patients and $\mathrm{CNs}$ (Fig. 6A-K). To investigate whether internalized $A \beta_{1-42}$ was effectively digested by monocytes in $\mathrm{AD}$, we tested the expression of cathepsin $\mathrm{D}$ and cathepsin $\mathrm{S}$, two main lysosomal aspartic and cysteine proteases, which were suggested to mediate $A \beta_{1-42}$ degradation $^{24-26}$. There were no significant differences in the protein levels of $A \beta$-degrading enzymes, including cathepsin $\mathrm{D}$ and cathepsin $\mathrm{S}$, between $\mathrm{AD}$ patients and $\mathrm{CNs}$ (Fig. 6L-M).

\section{Discussion}

Increasing evidence suggests that failure of $A \beta$ clearance leads to accumulation of $A \beta$ plaques over 20-30 years prior 

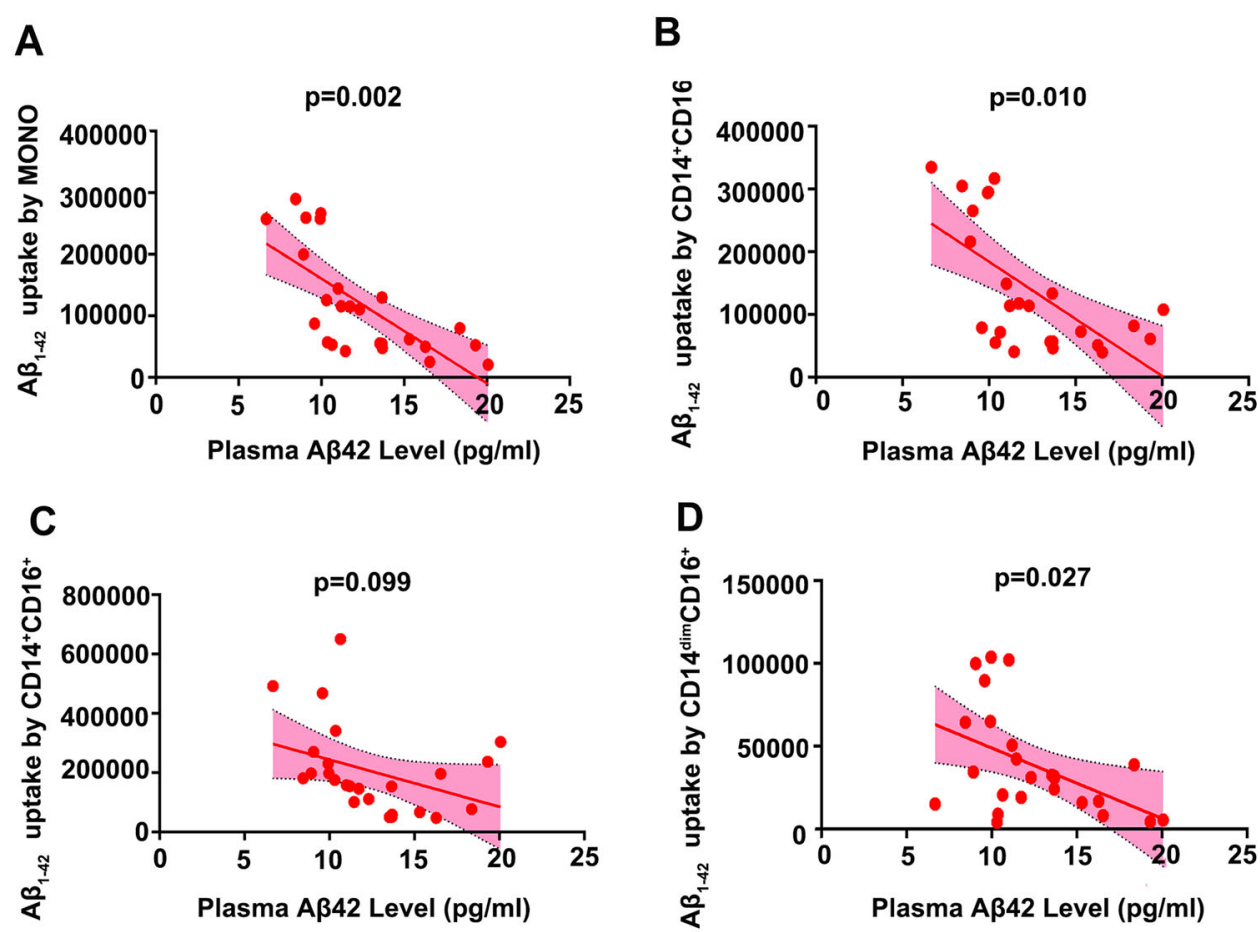

Fig. 3 Association of monocyte $\mathbf{A} \boldsymbol{\beta}_{1-42}$ uptake with plasma $\mathbf{A} \boldsymbol{\beta}_{1-42}$ levels in $\mathbf{C N}$ subjects. Correlations between plasma $A \beta_{1-42}$ and the uptake of $A \beta_{1-42}$ by all monocytes $(\mathbf{A})$, by the $C D 14^{+} C D 16^{-}$subset $(\mathbf{B})$, by the $C D 14^{+} C D 16^{+}$subset $(\mathbf{C})$ and by the $C D 14^{\operatorname{dim}} C D 16^{-}$subset $(\mathbf{D}) . n=25$, covariate correlation analysis. MONO monocytes, $A \beta$ amyloid- $\beta$ protein.
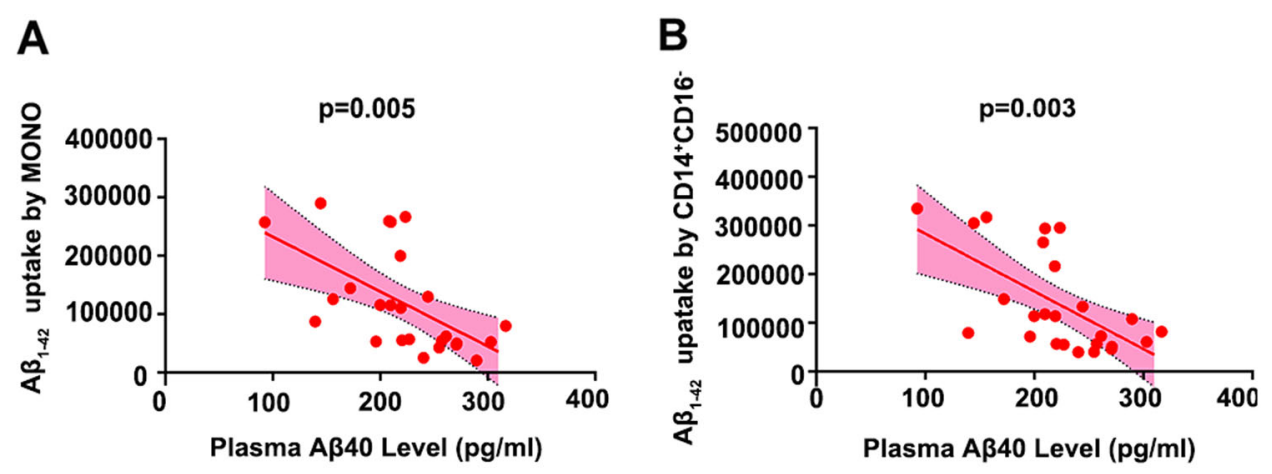

C

D
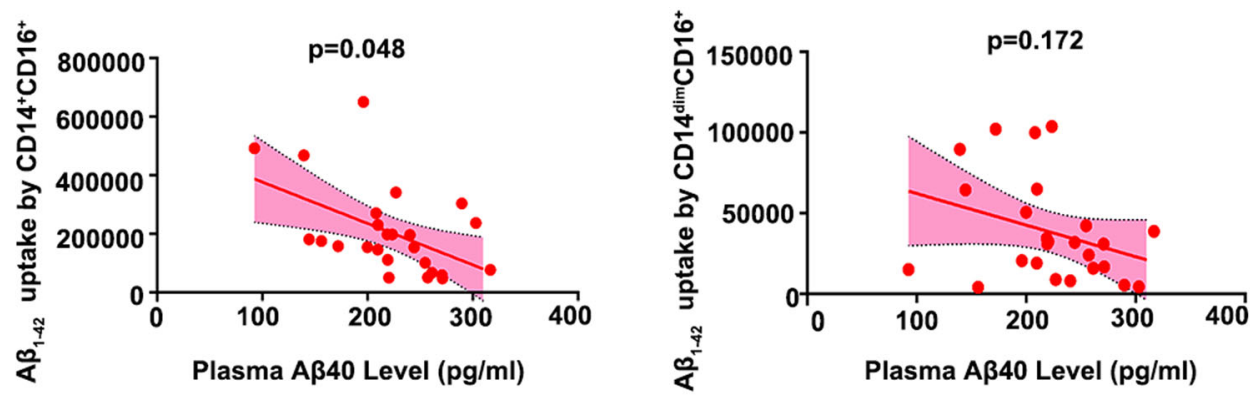

Fig. 4 Association of monocyte $\mathbf{A} \boldsymbol{\beta}_{1-42}$ uptake with plasma $\mathbf{A} \boldsymbol{\beta}_{1-40}$ levels in $\mathbf{C N}$ subjects. Correlations between plasma $A \beta_{1-42}$ and the uptake of $A \beta_{1-42}$ by all monocytes $(\mathbf{A})$, by the $C D 14^{+} C D 16^{-}$subset $(\mathbf{B})$, by the $C D 14^{+} C D 16^{+}$subset $(\mathbf{C})$ and by the $C D 14^{\operatorname{dim}} C D 16^{-}$subset $(\mathbf{D}) . n=25$, covariate correlation analysis. MONO monocytes, $A \beta$ amyloid- $\beta$ protein. 

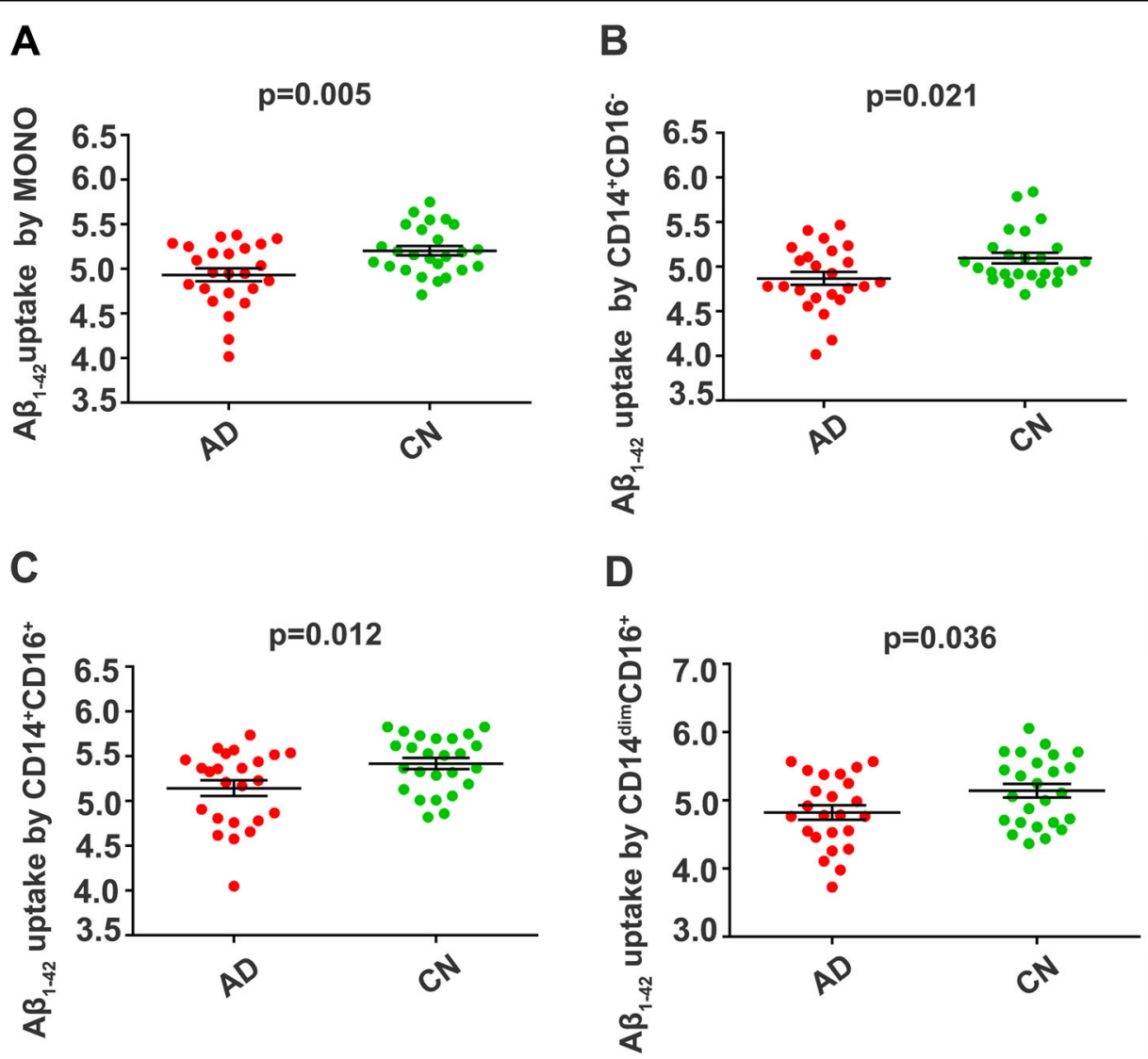

Fig. 5 Comparison of $A \beta_{1-42}$ uptake by monocyte subsets between $A D$ patients and $C N$ subjects. Compared with $C N$ controls, $A D$ patients had decreased $A \beta_{1-42}$ uptake by total monocytes $(\mathbf{A})$, the $C D 14^{+} \mathrm{CD} 16^{-}$subset $(\mathbf{B})$, the $\mathrm{CD} 14^{+} \mathrm{CD} 16^{+}$subset $(\mathbf{C})$ and the $\mathrm{CD} 14^{\mathrm{dim}} \mathrm{CD} 16^{-}$subset $(\mathbf{D}) . n=24$ for $A D ; n=25$ for $C N$, mean \pm SEM, Student's $t$ test, two sided. AD Alzheimer's disease, CN cognitively normal control, MONO monocytes, A 3 amyloid$\beta$ protein.

to the onset of dementia ${ }^{27,28}$. A previous study has demonstrated ineffective uptake of $\mathrm{A} \beta$ in $\mathrm{AD}$ patients. However, whether there exist specific alterations of $A \beta$ uptake in different monocyte subsets and the role of peripheral monocytes in $\mathrm{A} \beta$ uptake in $\mathrm{AD}$ remains unclear. Ageing is an important factor for the development of $\mathrm{AD}^{29}$. We found that $A \beta_{1-42}$ uptake by monocytes decreased as age increased. Consistently, a previous study showed that the internalization of $A \beta_{1-42}$ by aged human blood cell-derived monocytes was lower than that by human umbilical cord blood cell-derived monocytes ${ }^{30}$. But it did not show the trajectories of $A \beta$ uptake by different monocyte subsets during ageing. In our study, it is worth noting that the decrease in $A \beta$ uptake by classic monocyte subset and non-classic subset began at the age of 20 years and 40 years respectively, suggesting that the decrease in $\mathrm{A} \beta$ uptake ability is a life-long process that may be different among monocyte subsets and occurs prior to brain $A \beta$ deposition. Despite the impact of ageing, the $A \beta$ uptake ability of monocytes is further decreased in $A D$ patients, implying that compromised $\mathrm{A} \beta$ uptake by monocytes is involved in AD pathogenesis ${ }^{31-33}$.
In humans, peripheral monocytes can be divided into non-classic, intermediate and classic monocyte subsets ${ }^{23}$. These monocyte subsets may have different functions in AD. As reflected by our findings, the intermediate subset had the highest $A \beta$ uptake ability, while there was no significant difference in $A \beta$ uptake between the classic and non-classic subsets in $\mathrm{CN}$ controls. The $\mathrm{A} \beta$ uptake ability was mainly decreased in the classic and non-classic subsets during ageing, except for the most effective intermediate subset. However, $A \beta$ uptake ability was decreased in all subsets in AD patients. A previous study reports that $\mathrm{CD} 14^{+} \mathrm{CD} 16^{+}$subset is the main producer of interleukin (IL)-10 upon stimulation of TLRs ${ }^{34}$. However, dysfunctional $\mathrm{CD} 14^{+} \mathrm{CD} 16^{+}$subset not only affect $\mathrm{A} \beta$ clearance in $\mathrm{AD}$ but also may lead to reduction of IL-10, which is an anti-inflammatory cytokine and protective factor for neurogenesis and cognitive preservation, thus exacerbating the pathogenesis of $\mathrm{AD}^{14}$. These results suggest that there is an overall decrease in $A \beta$ uptake by all three monocyte subsets in AD patients, implying that the mechanisms underlying the alteration in $A \beta$ uptake 


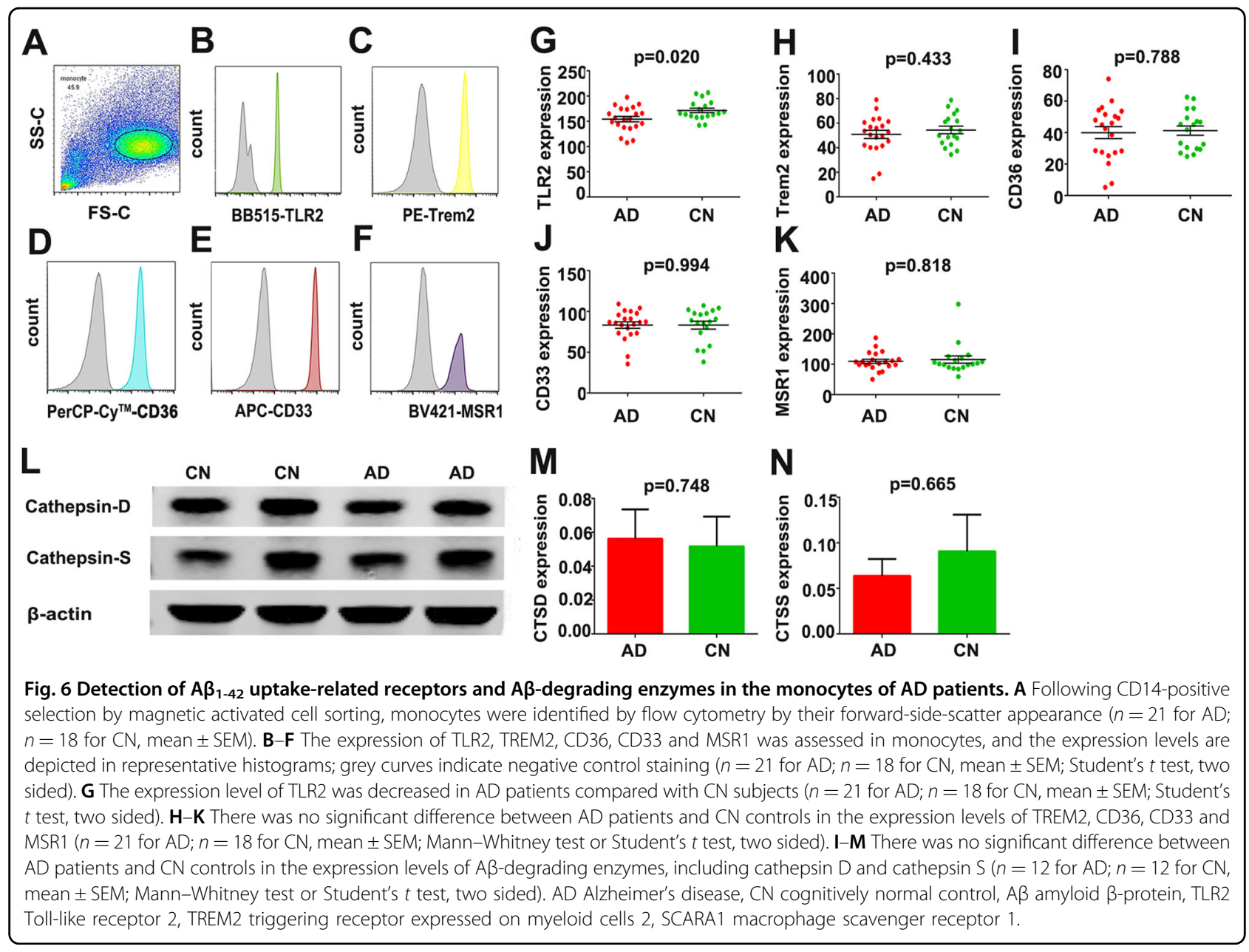

ability by monocytes in $\mathrm{AD}$ patients are different from those associated with ageing.

The mechanisms underlying the decreased $A \beta$ uptake ability by monocytes during ageing and $\mathrm{AD}$ remain to be investigated. We found that the decrease of $A \beta$ uptake could be partially due to deficits in $A \beta$ recognition by monocytes, as reflected by the reduced expression of TRL2 in monocytes. TRL2 belongs to a type I transmembrane pattern recognition receptor and acts as a natural innate immune receptor for $A \beta$ uptake through the formation of a receptor complex with CD14. Deletion or inhibition of the CD14-TLR receptor complex will impair fibrillary $A \beta_{1-42}$ uptake in human monocytes and delay cognitive decline in a mouse model of $\mathrm{AD}^{35,36}$. Previous studies have explored the intracellular processing and degradation of $A \beta$ within monocyte. After receptor-mediated $A \beta$ endocytosis, early endosomes are formed and further combined with lysosomes for degradation ${ }^{37}$. Thereinto, cathepsin D and cathepsin $S$, which are the two main lysosomal aspartic and cysteine proteases, were demonstrated to mediate $A \beta$ degradation. However, we did not find any differences in the expression of these two $A \beta$-degrading enzymes between $A D$ patients and $C N$ controls, suggesting that dysfunctional $A \beta$ recognition could be particularly important for the decrease in $A \beta$ uptake by monocytes in $\mathrm{AD}$ patients.

The decrease in $A \beta$ uptake by monocytes seems specific to $\mathrm{AD}$, as it was not changed in PD patients compared with $\mathrm{CN}$ controls in our study. Additional evidence indicates that the $A \beta$ uptake ability is correlated with blood $A \beta$ levels in $C N$ subjects, that is, the greater the $A \beta$ uptake ability is, the lower the blood $A \beta$ levels. These findings suggest that monocytes might play a critical role in clearing $A \beta$ from the blood.

In conclusion, our findings are of significance to the understanding of the pathogenesis of sporadic $\mathrm{AD}$. $\mathrm{A} \beta$ in the brain can be transported to the peripheral blood ${ }^{38-40}$, and the clearance of $A \beta$ in the periphery has been suggested to substantially contribute to the clearance of $A \beta$ from the brain ${ }^{10,41}$. Therefore, the decrease in $A \beta$ uptake by monocytes could play a significant role in the development of sporadic $\mathrm{AD}$. The recovery of the $\mathrm{A} \beta$ uptake function of blood monocytes may represent a therapeutic strategy for 
AD. The potential methods include adoptive transfer of healthy monocytes and bone marrow transplantation, modification of monocyte with $A \beta$-degrading enzymes and activation of monocytes by cytokines or agents ${ }^{42}$.

\section{Acknowledgements}

The authors would like to thank the patients and their families for their participation and dedication to research. This study was supported by National Natural Science Foundation of China (81930028, 91749206, 81625007 and 31921003)

\section{Author details}

'Department of Neurology and Centre for Clinical Neuroscience, Daping Hospital, Third Military Medical University, Chongqing, China. ${ }^{2}$ The Institute of Brain and Intelligence, Third Military Medical University, Chongqing, China. ${ }^{3}$ Chongqing Key Laboratory of Ageing and Brain Diseases, Chongqing, China. ${ }^{4}$ Department of Health Management, Daping Hospital, Third Military Medical University, Chongqing, China. ${ }^{5}$ State Key Laboratory of Trauma, Burn and Combined Injury, Daping Hospital, Third Military Medical University, Chongqing, China. ${ }^{6}$ Center for Excellence in Brain Science and Intelligence Technology, Chinese Academy of Sciences, Shanghai, China

\section{Author contributions}

Y.-J.W., Y.C., S.-H.C. and D.-Y.T. conceived and designed the project; Y.-Y.S. X.-L.B., J.L. and F.Z. collected human sample and neuropsychological battery, S.-H.C., D.-Y.T., Y.C., H.-L.S., C.-Y.H. and P.-Y.S. performed human sample experiments, S.-H.C., D.-Y.T., D.-Y.F. and Z.-Q.X. analysed data. Y.-J.W., Y.C., S.-H.C. and D.-Y.T. wrote and revised the manuscript. All authors read and approved the content of this manuscript.

\section{Conflict of interest}

The authors declare that they have no conflict of interest.

\section{Publisher's note}

Springer Nature remains neutral with regard to jurisdictional claims in published maps and institutional affiliations.

Supplementary Information accompanies this paper at (https://doi.org/ 10.1038/s41398-020-01113-9).

Received: 17 July 2020 Revised: 7 November 2020 Accepted: 17 November 2020

Published online: 08 December 2020

\section{References}

1. Prince, $M$. et al. The global prevalence of dementia: a systematic review and metaanalysis. Alzheimers Dement. 9, 63-75.e62 (2013).

2. Mawuenyega, K. G. et al. Decreased clearance of CNS beta-amyloid in Alzheimer's disease. Science 330, 1774 (2010).

3. Bradshaw, E. M. et al. CD33 Alzheimer's disease locus: altered monocyte function and amyloid biology. Nat. Neurosci. 16, 848-850 (2013).

4. Carmona, S. et al. The role of TREM2 in Alzheimer's disease and other neurodegenerative disorders. Lancet Neurol. 17, 721-730 (2018).

5. Vasquez, J. B., Fardo, D. W. \& Estus, S. ABCA7 expression is associated with Alzheimer's disease polymorphism and disease status. Neurosci. Lett. 556 58-62 (2013).

6. Crehan, H., Hardy, J. \& Pocock, J. Blockage of CR1 prevents activation of rodent microglia. Neurobiol. Dis. 54, 139-149 (2013).

7. Kunkle, B. W. et al. Genetic meta-analysis of diagnosed Alzheimer's disease identifies new risk loci and implicates $A \beta$, tau, immunity and lipid processing. Nat. Genet. 51, 414-430 (2019).

8. Krabbe, G. et al. Functional impairment of microglia coincides with Betaamyloid deposition in mice with Alzheimer-like pathology. PLOS ONE 8, e60921-e60921 (2013).

9. Grathwohl, S. A. et al. Formation and maintenance of Alzheimer's disease beta-amyloid plaques in the absence of microglia. Nat. Neurosci. 12, 1361-1363 (2009).
10. Xiang, Y. et al. Physiological amyloid-beta clearance in the periphery and its therapeutic potential for Alzheimer's disease. Acta Neuropathol. 130, 487-499 (2015).

11. Yuede, C. M. et al. Rapid in vivo measurement of $\beta$-amyloid reveals biphasic clearance kinetics in an Alzheimer's mouse model. J. Exp. Med. 213, 677-685 (2016).

12. Qosa, $H$. et al. Differences in amyloid- $\beta$ clearance across mouse and human blood-brain barrier models: kinetic analysis and mechanistic modeling. Neuropharmacology 79, 668-678 (2014).

13. Butovsky, O., Kunis, G., Koronyo-Hamaoui, M. \& Schwartz, M. Selective ablation of bone marrow-derived dendritic cells increases amyloid plaques in a mouse Alzheimer's disease model. Eur. J. Neurosci. 26, 413-416 (2007).

14. Koronyo-Hamaoui, M. et al. Attenuation of AD-like neuropathology by harnessing peripheral immune cells: local elevation of IL-10 and MMP-9. J. Neurochem. 111, 1409-1424 (2009).

15. Koronyo, Y. et al. Therapeutic effects of glatiramer acetate and grafted CD115 (+) monocytes in a mouse model of Alzheimer's disease. Brain 138, 2399-2422 (2015).

16. Simard, A. R., Soulet, D., Gowing, G., Julien, J. P. \& Rivest, S. Bone marrowderived microglia play a critical role in restricting senile plaque formation in Alzheimer's disease. Neuron 49, 489-502 (2006).

17. Hawkes, C. A. \& McLaurin, J. Selective targeting of perivascular macrophages for clearance of beta-amyloid in cerebral amyloid angiopathy. Proc. Natl Acad. Sci. USA 106, 1261-1266 (2009).

18. Li, W. W. et al. Association of polygenic risk score with age at onset and cerebrospinal fluid biomarkers of Alzheimer's disease in a Chinese cohort. Neurosci. Bull. 36, 696-704 (2020).

19. Hughes, A. J., Daniel, S. E., Kilford, L. \& Lees, A. J. Accuracy of clinical diagnosis of idiopathic Parkinson's disease: a clinico-pathological study of 100 cases. J. Neurol. Neurosurg. Psychiatry 55, 181-184 (1992).

20. Qian, F. \& Montgomery, R. R. Imaging immunosenescence. Methods Mol. Biol. 1343, 97-106 (2015).

21. Wilke, C. et al. Serum neurofilament light is increased in multiple system atrophy of cerebellar type and in repeat-expansion spinocerebellar ataxias: a pilot study. J. Neurol. 265, 1618-1624 (2018).

22. Lue, L. F., Guerra, A. \& Walker, D. G. Amyloid beta and tau as Alzheimer's disease blood biomarkers: promise from new technologies. Neurol. Ther. 6, 25-36 (2017).

23. Passlick, B., Flieger, D. \& Ziegler-Heitbrock, H. W. Identification and characterization of a novel monocyte subpopulation in human peripheral blood. Blood 74, 2527-2534 (1989)

24. Liuzzo, J. P., Petanceska, S. S. \& Devi, L. A. Neurotrophic factors regulate cathepsin $S$ in macrophages and microglia: a role in the degradation of myelin basic protein and amyloid beta peptide. Mol. Med. 5, 334-343 (1999).

25. Munger, J. S. et al. Lysosomal processing of amyloid precursor protein to $\mathrm{A}$ beta peptides: a distinct role for cathepsin S. Biochem. J. 311(Pt 1), 299-305 (1995).

26. Hamazaki, H. Cathepsin D is involved in the clearance of Alzheimer's betaamyloid protein. FEBS Lett. 396, 139-142 (1996).

27. Masters, C. L. et al. Alzheimer's disease. Nat. Rev. Dis. Prim. 1, 15056 (2015).

28. Villemagne, $V$. L. et al. Amyloid $\beta$ deposition, neurodegeneration, and cognitive decline in sporadic Alzheimer's disease: a prospective cohort study. Lancet Neurol. 12, 357-367 (2013).

29. Hou, Y. et al. Ageing as a risk factor for neurodegenerative disease. Nat. Rev Neurol. 15, 565-581 (2019).

30. Darlington, D. et al. Human umbilical cord blood-derived monocytes improve cognitive deficits and reduce amyloid-beta pathology in PSAPP mice. Cell Transpl. 24, 2237-2250 (2015).

31. Avagyan, H. et al. Immune blood biomarkers of Alzheimer disease patients. J. Neuroimmunol. 210, 67-72 (2009).

32. Fiala, M. et al. Ineffective phagocytosis of amyloid-beta by macrophages of Alzheimer's disease patients. J. Alzheimers Dis. 7, 221-232 (2005).

33. Gu, B. J. et al. Innate phagocytosis by peripheral blood monocytes is altered in Alzheimer's disease. Acta Neuropathol. 132, 377-389 (2016).

34. Skrzeczyńska-Moncznik, J. et al. Peripheral blood CD14high CD16+ monocytes are main producers of IL-10. Scand. J. Immunol. 67, 152-159 (2008)

35. Richard, K. L., Filali, M., Préfontaine, P. \& Rivest, S. Toll-like receptor 2 acts as a natural innate immune receptor to clear amyloid beta 1-42 and delay the 
cognitive decline in a mouse model of Alzheimer's disease. J. Neurosci. 28 5784-5793 (2008).

36. Udan, M. L., Ajit, D., Crouse, N. R. \& Nichols, M. R. Toll-like receptors 2 and 4 mediate Abeta(1-42) activation of the innate immune response in a human monocytic cell line. J. Neurochem. 104, 524-533 (2008).

37. Koronyo-Hamaoui, M. et al. Peripherally derived angiotensin converting enzyme-enhanced macrophages alleviate Alzheimer-related disease. Brain 143, 336-358 (2020).

38. Maness, L. M., Banks, W. A., Podlisny, M. B., Selkoe, D. J. \& Kastin, A. J. Passage of human amyloid beta-protein 1-40 across the murine blood-brain barrier. Life Sci. 55, 1643-1650 (1994).
39. Roberts, K. F. et al. Amyloid-beta efflux from the central nervous system into the plasma. Ann. Neurol. 76, 837-844 (2014).

40. Deane, R., Bell, R. D., Sagare, A. \& Zlokovic, B. V. Clearance of amyloid-beta peptide across the blood-brain barrier: implication for therapies in Alzheimer's disease. CNS Neurol. Disord. Drug Targets 8, 16-30 (2009).

41. Wang, J., Gu, B. J., Masters, C. L. \& Wang, Y. J. A systemic view of Alzheimer disease - insights from amyloid-beta metabolism beyond the brain. Nat. Rev. Neurol. 13, 612-623 (2017).

42. Zuroff, L., Daley, D., Black, K. L. \& Koronyo-Hamaoui, M. Clearance of cerebral Abeta in Alzheimer's disease: reassessing the role of microglia and monocytes. Cell Mol. Life Sci. 74, 2167-2201 (2017). 